\title{
ORAL MERCAPTOPURINE ADHERENCE IN PEDIATRIC ALL: A SURVEY STUDY FROM THE DFCI ALL CONSORTIUM
}

\author{
Justine Kahn ${ }^{1}$, Maneka Puligandla ${ }^{2}$, Kristen Stevenson ${ }^{3}$, Peter Cole ${ }^{4}$, Jennifer Welch ${ }^{5}$, \\ Melissa Beauchemin ${ }^{6}$, Elizabeth Gage-Bouchard ${ }^{7}$, Lewis Silverman ${ }^{2}$, Kara Kelly ${ }^{7}$, and Kira \\ Bona $^{3}$ \\ ${ }^{1}$ Columbia University Medical Center \\ ${ }^{2}$ Dana Farber Cancer Institute \\ ${ }^{3}$ Dana-Farber Cancer Institute \\ ${ }^{4}$ Rutgers The State University of New Jersey \\ ${ }^{5}$ Hasbro Children's Hospital \\ ${ }^{6}$ Columbia University Medical Centre \\ ${ }^{7}$ Roswell Park Comprehensive Cancer Center
}

October 20, 2020

\begin{abstract}
Poor adherence to 6-mercaptopurine during acute lymphoblastic leukemia (ALL) therapy increases relapse risk. Clinicallysignificant non-adherence has been documented in up to $30 \%$ of children treated for ALL on Children's Oncology Group trials. Whether non-adherence rates vary across regimens with different chemotherapy schedules and modes of administration is not known. In a cross-sectional survey study of $\mathrm{N}=61$ children treated on, or as per Dana-Farber Cancer Institute (DFCI) ALL Consortium Protocol 11-001, 25\% (95\% CI 14-37\%) of respondents self-reported non-adherence to $6 \mathrm{MP}$, suggesting that the frequency of non-adherence may be similar across different Consortia regimens.
\end{abstract}

\begin{abstract}
Poor adherence to 6-mercaptopurine during acute lymphoblastic leukemia (ALL) therapy increases relapse risk. Clinically-significant non-adherence has been documented in up to $30 \%$ of children treated for ALL on Children's Oncology Group trials. Whether non-adherence rates vary across regimens with different chemotherapy schedules and modes of administration is not known. In a cross-sectional survey study of $\mathrm{N}=61$ children treated on, or as per Dana-Farber Cancer Institute (DFCI) ALL Consortium Protocol 11-001, 25\% (95\% CI 14-37\%) of respondents self-reported non-adherence to 6MP, suggesting that the frequency of non-adherence may be similar across different Consortia regimens.
\end{abstract}

\section{INTRODUCTION}

Successful treatment of pediatric acute lymphoblastic leukemia (ALL) requires at least two-years of riskdirected therapy built on a backbone of oral chemotherapy, including 6-mercaptopurine (6MP) and dexamethasone. Studies from the Children's Oncology Group (COG) demonstrated that less than $90 \%$ adherence to $6 \mathrm{MP}$ during maintenance therapy was associated with up to a 3.9 -fold increased relapse risk. ${ }^{1}$ Non-adherence in these studies was associated with lack of routine surrounding medication taking, patient race/ethnicity and age, household structure, and parent educational attainment. ${ }^{1}$ Little is known about $6 \mathrm{MP}$ adherence rates in the context of non-COG ALL treatment regimens - where different treatment schedules and modes 
of chemotherapy delivery (e.g. oral versus intravenous [IV] administration of chemotherapy agents) may impact frequency and reasons for non-adherence.

Compared to the continuous daily $6 \mathrm{MP}$, oral weekly methotrexate and repeating 4-week cycles in COG ALL trials, Dana-Farber Cancer Institute (DFCI) ALL Consortium protocols utilize 14-day pulses of 6MP with weekly IV methotrexate throughout maintenance in repeating 3 -week cycles. ${ }^{2}$ This chemotherapy delivery approach requires more frequent contact between families and healthcare providers and reduces the number of oral chemotherapy agents. We explored whether the "higher-touch" of every 3-week clinic visits for cycle initiation along with use of IV methotrexate-necessitating weekly clinic visits or visiting nurse administration - might reduce the frequency of 6MP non-adherence on the DFCI regimen as compared to published COG data. ${ }^{3}$ Specifically, we aimed to explore the prevalence of parent-reported 6MP nonadherence in the context of DFCI ALL Consortium-based therapy utilizing a cross-sectional survey, and to identify whether sociodemographic factors, medication-taking logistics or chemotherapy comprehension impacted adherence rates in a DFCI cohort.

\section{Results}

\section{PATIENTS and METHODS}

Study Context: The DFCI ALL Consortium conducts Phase 3 clinical trials for ALL at 8 U.S. and Canadian centers. DFCI Protocol 11-001 enrolled children from 2012 to 2015, and treatment as per DFCI 11-001 became the standard of care for patients at participating Consortium sites following trial closure after accrual. DFCI 11-001 included a two-year treatment course with a 70-week Continuation (maintenance) phase. Each 3-week cycle included day 1 vincristine (IV), days $1-5$ dexamethasone (oral), days 1, 8, 15 methotrexate (IV), and days 1-14 6MP (oral). Parents were instructed to administer 6MP at bedtime, on an empty stomach, at least two hours before, and one hour after food or milk.

Patients: Eligible participants were English or Spanish speaking caregivers of children ages 1-18 years receiving Continuation therapy on or as per DFCI 11-001 between February 2015 and March 2017 at one of four participating Consortium sites (Montefiore Medical Center, Bronx, NY, Columbia University Irving Medical Center, New York, NY, DFCI, Boston, MA, and Hasbro Children's Hospital of Rhode Island, Providence, RI). Informed consent/assent was obtained for all subjects and the study was approved by the Institutional Review Board at participating sites.

Surveys: Parent/guardians completed a single time-point, paper-pencil survey during a regularly scheduled clinic visit on Day 1 of a 3-week Continuation cycle. The survey included sociodemographic variables (patient/parent age, race, ethnicity, country of origin, family structure, education for each parent/guardian, household income, food insecurity, and marital status $)^{4}$ as well as 33 dichotomous and multiple-choice questions related to 6MP comprehension, administration, and adherence adapted with permission from Bhatia et al. ${ }^{4}$ Low parental education was defined as having completed some high-school or less vs. high school graduate or more. ${ }^{5}$ Adherence survey questions inquired about medication-taking routines, daily administration challenges, and perceived risks associated with missing 6MP. Free-text questions asked whether more resources or reminders would be helpful to improve adherence.

Non-adherence: Patients were defined as 6MP non-adherent using two survey metrics of missed doses. These included: (1) a response of $<14$ days to the question "During the past 3 weeks, how many days did your child take 6MP?" and (2) endorsement of missed doses during a prior cycle, or other changes to prescribed chemotherapy without instruction from a medical provider.

\section{Statistical Analysis}

Descriptive statistics were generated for all data. Comparison between two groups (adherent and nonadherent) was performed using two-sided Wilcoxon rank-sum tests for continuous variables and Fisher exact tests for categorical variables. 95\% exact binomial confidence intervals were calculated for the proportion of patients considered adherent and additional survey questions. P-values $<0.05$ were considered significant 


\section{RESULTS}

The analytic cohort included 61 families with evaluable self-report adherence data (Table 1). Fifteen (25\%, [95\% CI, 14-37\%]) patients met the study definition of 6MP non-adherence. Twenty-five percent did not have a medication-taking routine. Paternal education level differed significantly between adherent and nonadherent patients. Of the group of fathers who completed high school or more $81 \%$ were adherent compared to only $43 \%$ who did not complete high school $(\mathrm{p}=0.045)$. Five percent of participants endorsed difficulty paying for their $6 \mathrm{MP}$, however this was not associated with non-adherence.

When asked "what would happen if your child stopped taking 6MP," only N= 30 (49\% [95\% CI, 36-62\%]) parents reported "relapse" or "cancer returning." The most common reasons for missing 6MP were related to dosing restrictions around food or falling asleep prior to bedtime dose administration. Fourteen (23\% [95\% CI 13-36\%]) parents reported that it was "not easy" to follow 6MP administration guidelines. Across the full cohort, $\mathrm{N}=24$ (39\% [95\% CI 27-53\%]) participants suggested that printed calendars would help with adherence and $\mathrm{N}=35$ (57\% [95\% CI, 44-70\%]) expressed interest in receiving educational resources about $6 \mathrm{MP}$

\section{DISCUSSION}

Among patients treated with a DFCI ALL Consortium regimen, 25\% [95\% CI, 14\% - 37\%] of caregivers report non-adherence to oral 6MP during therapy. This rate is strikingly similar to Medication Event Monitoring System (MEMS)-measured adherence rates from the COG. ${ }^{3,6}$ Given that self-reported non-adherence has a sensitivity as low as $53 \%$ but a positive predictive value nearing $95 \%,{ }^{7}$ these data suggest that the frequency of non-adherence in this cohort is likely an under-estimate of the true prevalence, and even higher than the non-adherence rates observed in the COG.

Similar to other studies, non-adherence was associated with parent education level, ${ }^{3}$ and barriers to adherence included 6MP administration guidelines surrounding food and dairy intake. Only $49 \%$ of parents recognized "relapse" or "cancer returning" as risks associated with non-adherence. Studies have demonstrated that patients who understand the risks and benefits of following medication guidelines are more likely to adhere to prescribed regimens. ${ }^{8}$ We previously reported significant variability in approaches to patient education surrounding oral chemotherapy across DFCI ALL Consortium sites. ${ }^{9}$ Specifically, providers reported modifying chemotherapy teaching based on their perceptions of patient comprehension. Our finding that $25 \%$ of families in the DFCI cohort reported non-adherence to 6MP suggests that more frequent patient-provider contact, and individualized oral chemotherapy education do not mitigate barriers to adherence.

This study was limited by its small sample size, cross-sectional nature, and self-report adherence measure. Despite limitations, these exploratory data demonstrate that 1 in 4 families self-report oral $6 \mathrm{MP}$ non-adherence in the context of a chemotherapy regimen reliant on weekly interactions with the medical team. These findings support the generalizable nature of published non-adherence data across pediatric oncology consortia, reinforcing the urgent need for healthcare delivery intervention development to address non-adherence. Given that parental education is associated with non-adherence across consortia, future interventions targeting the efficacy of medical communication may be warranted. A prospective investigation of oral chemotherapy adherence using a MEMS-based approach is ongoing in the current DFCI ALL Consortium trial 16-001 (NCT03020030).

\section{Acknowledgements:}

We would like to thank Dr. Smita Bhatia for sharing her expertise in adherence research as well as her permission to adapt her adherence survey instrument for this study.

JMK is supported by a National Institutes of Health KL2 award from the Irving Institute for Clinical and Translational Research at Columbia University Irving Medical Center (NIH-KL2TR001874)

MB is supported by a T32 award from The National Cancer Institute Training Grant (T32CA094061).

KB is supported by a K07 award from the National Institutes of Health (K07CA211847). 


\section{References}

1. Bhatia S, Landier W, Hageman L, et al. Systemic Exposure to Thiopurines and Risk of Relapse in Children With Acute Lymphoblastic Leukemia: A Children's Oncology Group Study. JAMA oncology.2015;1(3):287295.

2. Vrooman LM, Blonquist TM, Harris MH, et al. Refining risk classification in childhood B acute lymphoblastic leukemia: results of DFCI ALL Consortium Protocol 05-001. Blood Adv.2018;2(12):1449-1458.

3. Bhatia S, Landier W, Hageman L, et al. 6MP adherence in a multiracial cohort of children with acute lymphoblastic leukemia: a Children's Oncology Group study. Blood. 2014;124(15):2345-2353.

4. Bhatia S, Landier W, Shangguan M, et al. Nonadherence to oral mercaptopurine and risk of relapse in Hispanic and non-Hispanic white children with acute lymphoblastic leukemia: a report from the children's oncology group. J Clin Oncol. 2012;30(17):2094-2101.

5. US Census Bureau, American Community Survey. 2016.

6. Landier W, Hageman L, Chen Y, et al. Mercaptopurine Ingestion Habits, Red Cell Thioguanine Nucleotide Levels, and Relapse Risk in Children With Acute Lymphoblastic Leukemia: A Report From the Children's Oncology Group Study AALL03N1. Journal of clinical oncology : official journal of the American Society of Clinical Oncology.2017;35(15):1730-1736.

7. Landier W, Chen Y, Hageman L, et al. Comparison of self-report and electronic monitoring of $6 \mathrm{MP}$ intake in childhood ALL: a Children's Oncology Group study. Blood. 2017;129(14):1919-1926.

8. Landier W, Hughes CB, Calvillo ER, et al. A grounded theory of the process of adherence to oral chemotherapy in Hispanic and caucasian children and adolescents with acute lymphoblastic leukemia. $J$ Pediatr Oncol Nurs. 2011;28(4):203-223.

9. Kahn JM, Athale UH, Clavell LA, et al. How Variable Is Our Delivery of Information? Approaches to Patient Education About Oral Chemotherapy in the Pediatric Oncology Clinic. $J$ Pediatr Health Care.2017;31(1):e1-e6.

\section{Hosted file}

Final 6MP survey table_10-15-20.pdf available at https://authorea.com/users/368781/articles/ 487802-oral-mercaptopurine-adherence-in-pediatric-all-a-survey-study-from-the-dfci-allconsortium 